TAIWANESE JOURNAL OF MATHEMATICS

Vol. 14, No. 2, pp. 403-412, April 2010

This paper is available online at http://www.tjm.nsysu.edu.tw/

\title{
CLASS OF ANALYTIC FUNCTIONS ASSOCIATED WITH NEW MULTIPLIER TRANSFORMATIONS AND HYPERGEOMETRIC FUNCTION
}

\author{
Adriana Cătaş
}

\begin{abstract}
The purpose of the paper is to derive various properties and characteristics of certain subclass of analytic functions using multiplier transformations and the method of differential subordination.
\end{abstract}

\section{InTROduction AND Definitions}

Let $\mathcal{H}$ be the class of analytic functions in the open unit disc $U=\{z \in \mathbb{C}$ : $|z|<1\}$ and $\mathcal{H}[a, n]$ be the subclass of $\mathcal{H}$ consisting of functions of the form $f(z)=a+\sum_{k=n}^{\infty} a_{k} z^{k}$. Let $\mathcal{A}(n)$ denote the class of functions $f(z)$ normalized by

$$
f(z)=z+\sum_{k=n+1}^{\infty} a_{k} z^{k} \quad(n \in \mathbb{N}:=\{1,2,3, \ldots\})
$$

which are analytic in the open unit disc. In particular, we set $\mathcal{A}(1):=\mathcal{A}$.

For $f(z)$ given by $(1.1)$ and $g(z)$ given by $g(z)=z+\sum_{k=n+1}^{\infty} b_{k} z^{k}$ the Hadamard product (or convolution), $(f * g)(z)$ is defined, by $(f * g)(z):=z+$ $\sum_{k=n+1}^{\infty} a_{k} b_{k} z^{k}:=(g * f)(z)$.

If $f$ and $g$ are analytic in $U$, we say that $f$ is subordinate to $g$, written symbolically as $f \prec g$ or $f(z) \prec g(z), \quad(z \in U)$ if there exists a Schwarz function $w(z)$ in $U$, which is analytic in $U$ with $w(0)=0$ and $|w(z)|<1$ such that $f(z)=g(w(z)), z \in U$.

Received January 24, 2008, accepted May 22, 2008.

Communicated by H. M. Srivastava.

2000 Mathematics Subject Classification: 30C45.

Key words and phrases: Analytic functions, Differential subordination, Gauss hypergeometric function, Multiplier transformation. 
Lemma 1.1. [6]. Let $h$ be a convex function with $h(0)=a$ and let $\gamma \in \mathbb{C}^{*}$ be a complex with $\operatorname{Re} \gamma \geq 0$. If $p \in \mathcal{H}[a, n]$ and $p(z)+\frac{1}{\gamma} z p^{\prime}(z) \prec h(z)$ then $p(z) \prec q(z) \prec h(z)$, where

$$
q(z)=\frac{\gamma}{n z^{\gamma / n}} \int_{0}^{z} h(t) t^{(\gamma / n)-1} d t .
$$

The function $q$ is convex and is the best $(a, n)$ dominant.

We denote by $\mathcal{P}(\delta)$, the class of functions $\phi$ which belong to $\mathcal{H}[1, n]$ and satisfy the inequality $\operatorname{Re}(\phi(z))>\delta, \quad(0 \leq \delta<1, z \in U)$. It is known [7] that if $\phi_{i} \in \mathcal{P}\left(\delta_{i}\right),\left(0 \leq \delta_{i}<1, i=1,2\right)$, then $\left(\phi_{1} * \phi_{2}\right) \in \mathcal{P}\left(\delta_{3}\right)$ where $\delta_{3}=1-2\left(1-\delta_{1}\right)\left(1-\delta_{2}\right)$ and the bound $\delta_{3}$ is the best possible.

Lemma 1.2. [6] Let the function $\phi \in \mathcal{H}[1,1]$ be in the class $\mathcal{P}(\delta)$. Then

$$
\operatorname{Re}(\phi(z)) \geq 2 \delta-1+\frac{2(1-\delta)}{1+|z|}, \quad(0 \leq \delta<1, z \in U) .
$$

Lemma 1.3. [15]. For real or complex numbers $a$, $b$ and $c\left(c \notin \mathbb{Z}_{0}^{-}:=\right.$ $\{0,-1,-2, \ldots\})$, $\operatorname{Re} c>\operatorname{Re} b>0$ we have

$$
\begin{gathered}
\int_{0}^{1} t^{b-1}(1-t)^{c-b-1}(1-t z)^{-a} d t=\frac{\Gamma(b) \Gamma(c-b)}{\Gamma(c)} \cdot{ }_{2} F_{1}(a, b ; c ; z), \\
{ }_{2} F_{1}(a, b, c ; z)=(1-z)^{-a} \cdot{ }_{2} F_{1}\left(a, c-b ; c ; \frac{z}{z-1}\right),
\end{gathered}
$$

$$
\begin{gathered}
{ }_{2} F_{1}(a, b ; c ; z)={ }_{2} F_{1}(b, a ; c ; z) \\
(b+1){ }_{2} F_{1}(1, b ; b+1 ; z)=(b+1)+b z \cdot{ }_{2} F_{1}(1, b+1 ; b+2 ; z)
\end{gathered}
$$

Lemma 1.4. [12]. Let $\phi$ be analytic in $U$ with $\phi(0)=1$ and $\operatorname{Re}(\phi(z))>\frac{1}{2}$ in $U$. Then for any function $F$ analytic in $U$, the function $\phi * F$ takes values in the convex hull of the image of $U$ under $F$.

Lemma 1.5. [6]. Suppose that the function $\psi: \mathbb{C}^{2} \times \mathbb{C} \rightarrow \mathbb{C}$ satisfies the condition $\operatorname{Re} \psi(i \rho, \sigma ; z) \leq \delta$, for $\delta>0$ and $\rho, \sigma \leq-\frac{1}{2}\left(1+\rho^{2}\right)$. If $\varphi \in \mathcal{H}[1,1]$ is analytic in $U$ and $\operatorname{Re} \psi\left(\varphi(z), z \varphi^{\prime}(z) ; z\right)>\delta$ then $\operatorname{Re} \varphi(z)>0$ in $U$. 
We propose

Definition 1.1. Let $f \in \mathcal{A}(n)$. For $m \in \mathbb{N}_{0}=\mathbb{N} \cup\{0\}, \lambda \geq 0, l \geq 0$ we define the multiplier transformations $I^{m}(\lambda, l)$ on $\mathcal{A}(n)$ by the following infinite series

$$
I^{m}(\lambda, l) f(z):=z+\sum_{k=n+1}^{\infty}\left[\frac{1+\lambda(k-1)+l}{1+l}\right]^{m} a_{k} z^{k} .
$$

It follows from (1.8) that

$$
\begin{gathered}
I^{0}(\lambda, l) f(z)=f(z) \\
(1+l) I^{2}(\lambda, l) f(z)=(1-\lambda+l) I^{1}(\lambda, l) f(z)+\lambda z\left(I^{1}(\lambda, l) f(z)\right)^{\prime} \\
I^{m_{1}}(\lambda, l)\left(I^{m_{2}}(\lambda, l) f(z)\right)=I^{m_{2}}(\lambda, l)\left(I^{m_{1}}(\lambda, l) f(z)\right)
\end{gathered}
$$

for all integers $m_{1}$ and $m_{2}$.

Remark 1.1. For $l=0, \lambda \geq 0$, the operator $D_{\lambda}^{m}:=I^{m}(\lambda, 0)$ was introduced and studied by Al-Oboudi [1] which reduces to the Salagean differential operator for $\lambda=1$ [11]. The operator $I_{l}^{m}:=I^{m}(1, l)$ was studied recently by Cho and Srivastava [2] and Cho and Kim [3]. The operator $I_{m}:=I^{m}(1,1)$ was studied by Uralegaddi and Somanatha [14].

\section{INCLUSION RESULTS}

Now we define a new class of analytic functions by using the multiplier transformations $I^{m}(\lambda, l)$ defined by (1.8) as follows.

Definition 2.2. Let $m \in \mathbb{N}_{0}=\mathbb{N} \cup\{0\}, A, B, \eta, \lambda, l$ be arbitrary fixed real numbers such that $-1 \leq B<A \leq 1, \eta \geq 0, \lambda \geq 0$ and $l \geq 0$. A function $f \in \mathcal{A}$ is said to be in the class $R_{\lambda}^{m}(\eta ; A, B)$ if it satisfies the following subordination

$$
\left(I^{m}(\lambda, l) f(z)\right)^{\prime}+\eta z\left(I^{m}(\lambda, l) f(z)\right)^{\prime \prime} \prec \frac{1+A z}{1+B z}, \quad(z \in U) .
$$

The class $R_{\lambda}^{m}(\eta ; A, B)$ generalizes a number of function classes studied earlier by several authors (see, e.g., Mac Gregor [5], Ponnusamy [10], Al-Oboudi [1] and Patel [8]). We write $\mathcal{R}_{\lambda}^{m}(0 ; 1-2 \alpha,-1) \equiv \mathcal{R}^{m}(1-2 \alpha,-1)$, the class of functions $f \in \mathcal{A}$ which satisfy the condition $\operatorname{Re}\left(I^{m}(\lambda, l) f(z)\right)^{\prime}>\alpha$. 
Theorem 2.1. We have $\mathcal{R}_{\lambda}^{m+1}(0 ; A, B) \subset \mathcal{R}_{\lambda}^{m}(1-2 \beta,-1)$ where $\beta$ is given by

$$
\beta= \begin{cases}\frac{A}{B}+\left(1-\frac{A}{B}\right)(1-B)_{2}^{-1} F_{1}\left(1,1 ; \frac{1+l}{\lambda n}+1 ; \frac{B}{B-1}\right), & B \neq 0 \\ 1-\frac{1+l}{1+l+\lambda n} A, & B=0\end{cases}
$$

The result is the best possible.

Proof. Setting $\varphi(z):=\left(I^{m}(\lambda, l) f(z)\right)^{\prime}$, we note that $\varphi \in \mathcal{H}[1, n]$. Making use the identity

$$
(1+l) I^{m+1}(\lambda, l) f(z)=(1-\lambda+l) I^{m}(\lambda, l) f(z)+\lambda z\left(I^{m}(\lambda, l) f(z)\right)^{\prime}
$$

we obtain

$$
\left(I^{m+1}(\lambda, l) f(z)\right)^{\prime}=\varphi(z)+\frac{z \varphi^{\prime}(z)}{(1+l) / \lambda} \prec \frac{1+A z}{1+B z}, \quad(z \in U) .
$$

Thus, by Lemma 1.1 for $\gamma=\frac{1+l}{\lambda}$, we deduce that

$$
\begin{aligned}
& \left(I^{m}(\lambda, l) f(z)\right)^{\prime} \prec q(z)=\frac{1+l}{\lambda n} z^{-\frac{1+l}{\lambda n}} \int_{0}^{z} t^{\frac{1+l}{\lambda n}-1} \cdot \frac{1+A t}{1+B t} d t \\
& = \begin{cases}\frac{A}{B}+\left(1-\frac{A}{B}\right)(1+B z)^{-1}{ }_{2} F_{1}\left(1,1 ; \frac{1+l}{\lambda n}+1 ; \frac{B z}{B z+1}\right), & B \neq 0 \\
1+\frac{1+l}{1+l+\lambda n} A z, & B=0\end{cases}
\end{aligned}
$$

where we have also made a change of variables followed by the use of the identities (1.4) and (1.5). Following the same lines as in Theorem 4 [9], we can prove that $\inf _{z \in U}\{\operatorname{Re} q(z)\}=q(-1)$. The proof of Theorem 2.1 is thus completed.

Remark 2.2. Theorem 2.1 improves the result obtained by Patel [[8], Theorem 2]. For $l=0, n=1, A=1-2 \alpha,(0 \leq \alpha<1)$ and $B=-1$ in Theorem 2.1, one obtains a result which also improves the corresponding work of Al-Oboudi [[1], Theorem 2.4].

\section{Convolution Properties}

Theorem 3.1. Let $-1 \leq B_{j}<A_{j} \leq 1,(j=1,2)$. If the functions $f_{j} \in$ $\mathcal{R}_{\lambda}^{m}\left(\eta ; A_{j}, B_{j}\right)(j=1,2)$, then the function $h \in \mathcal{A}$ defined by 


$$
h(z)=I^{m}(\lambda, l)\left(f_{1} * f_{2}\right)(z), \quad(z \in U)
$$

belongs to the class $\mathcal{R}_{\lambda}^{m}(\eta ; 1-2 \delta,-1)$, where

$$
\begin{gathered}
\delta=\sigma_{3}+(1-\eta)\left(1-\sigma_{3}\right)\left[{ }_{2} F_{1}\left(1,2 ; \frac{1}{n}+1 ; \frac{1}{2}\right)-1\right] \\
\sigma_{3}=1-2\left(1-\sigma_{1}\right)\left(1-\sigma_{2}\right) \\
\sigma_{j}=\left\{\begin{array}{ll}
\frac{A_{j}}{B_{j}}+\left(1-\frac{A_{j}}{B_{j}}\right)\left(1-B_{j}\right)^{-1} \cdot{ }_{2} F_{1}\left(1,1 ; \frac{1}{n \eta}+1 ; \frac{B_{j}}{B_{j}-1}\right), & B_{j} \neq 0 \\
1-\frac{1}{1+n \eta} A, & B_{j}=0
\end{array} .\right.
\end{gathered}
$$

Proof. Setting $\varphi_{j}(z)=\left(I^{m}(\lambda, l) f_{j}(z)\right)^{\prime}, \quad(z \in U)$ we note that $\varphi_{j}(z)$ belongs to the class $\mathcal{H}[1, n]$ and is analytic in $U$ for each $j=1,2$. Since $f_{j} \in \mathcal{R}_{\lambda}^{m}\left(\eta ; A_{j}, B_{j}\right)$ one obtains that

$$
\varphi_{j}(z)+\eta z \varphi_{j}^{\prime}(z)=\left(I^{m}(\lambda, l) f_{j}(z)\right)^{\prime}+\eta z\left(I^{m}(\lambda, l) f_{j}(z)\right)^{\prime \prime} \prec \frac{1+A_{j} z}{1+B_{j} z} .
$$

By making use of Lemma 1.1, with $\gamma=\frac{1}{\eta}$ and following the steps of proof of Theorem 2.1, we get $\left(I^{m}(\lambda, l) f_{j}(z)\right)^{\prime} \in \mathcal{P}\left(\sigma_{j}\right)$, for $j=1,2$ where

$$
\sigma_{j}=\left\{\begin{array}{ll}
\frac{A_{j}}{B_{j}}+\left(1-\frac{A_{j}}{B_{j}}\right)\left(1-B_{j}\right)^{-1} \cdot{ }_{2} F_{1}\left(1,1 ; \frac{1}{n \eta}+1 ; \frac{B_{j}}{B_{j}-1}\right), & B_{j} \neq 0 \\
1-\frac{1}{1+\eta n} A, & B_{j}=0
\end{array} .\right.
$$

Thus, for $h=I^{m}(\lambda, l)\left(f_{1} * f_{2}\right)(z)$ we have

$$
\left[z\left(I^{m}(\lambda, l) h(z)\right)^{\prime}\right]^{\prime}=\left(I^{m}(\lambda, l) f_{1}(z)\right)^{\prime} *\left(I^{m}(\lambda, l) f_{2}(z)\right)^{\prime} \in \mathcal{P}\left(\sigma_{3}\right),
$$

where $\sigma_{3}=1-2\left(1-\sigma_{1}\right)\left(1-\sigma_{2}\right)$ and

$$
\begin{aligned}
{\left[z\left(I^{m}(\lambda, l) h(z)\right)^{\prime}\right]^{\prime}=} & \left(I^{m}(\lambda, l) h(z)\right)^{\prime}+z\left(I^{m}(\lambda, l) h(z)\right)^{\prime \prime} \in \mathcal{P}\left(\sigma_{3}\right), \\
& \left(I^{m}(\lambda, l) h(z)\right)^{\prime} \in \mathcal{P}\left(\sigma_{4}\right)
\end{aligned}
$$

where $\sigma_{4}=\sigma_{3}+\left(1-\sigma_{3}\right)\left[{ }_{2} F_{1}\left(1,1 ; \frac{1}{n}+1 ; \frac{1}{2}\right)-1\right]$ is obtained by using Lemma 1.1 with $\gamma=1, A=1-2 \sigma_{3}$ and $B=-1$. 
It follows

$$
\begin{aligned}
& \operatorname{Re}\left[\left(I^{m}(\lambda, l) h(z)\right)^{\prime}+\eta z\left(I^{m}(\lambda, l) h(z)\right)^{\prime \prime}\right] \\
> & (1-\eta) \sigma_{4}+\eta \sigma_{3}=\sigma_{3}+(1-\eta)\left(1-\sigma_{3}\right)\left[{ }_{2} F_{1}\left(1,1 ; \frac{1}{n}+1 ; \frac{1}{2}\right)-1\right]=\delta .
\end{aligned}
$$

This completes the proof of Theorem 3.1.

Remark 3.3. Putting $A_{1}=A_{2}=1-2 \alpha,(0 \leq \alpha<1), B_{1}=B_{2}=-1$, $m=0$, and $\eta=2$ in Theorem 3.1, one improves a result obtained by Patel [8].

Theorem 3.2. Let $-1 \leq B_{j}<A_{j}<1(j=1,2)$. If the functions $f_{j} \in$ $\mathcal{R}_{\lambda}^{m}\left(0 ; A_{j}, B_{j}\right)(j=1,2)$, then the function $h$ defined by (3.1) belongs to the class $\mathcal{R}_{\lambda}^{m}(1-2 \rho,-1)$ where

$$
\begin{gathered}
\rho=2 \sigma_{3}-1+\left(1-\sigma_{3}\right){ }_{2} F_{1}\left(1,1 ; \frac{1}{n}+1 ; \frac{1}{2}\right) \\
\sigma_{3}=1-2 \frac{\left(A_{1}-B_{1}\right)\left(A_{2}-B_{2}\right)}{\left(1-B_{1}\right)\left(1-B_{2}\right)} .
\end{gathered}
$$

The result is the best possible for $B_{1}=B_{2}=-1$.

Proof. For each function $\varphi_{j}, j=1,2$ defined by $\varphi_{j}(z)=\left(I^{m}(\lambda, l) f_{j}(z)\right)^{\prime}$, we have $\varphi_{j} \in \mathcal{P}\left(\sigma_{j}\right), \sigma_{j}=\frac{1-A_{j}}{1-B_{j}}, j=1,2$ and $\varphi_{1} * \varphi_{2} \in \mathcal{P}\left(\sigma_{3}\right)$ where

$$
\sigma_{3}=1-2 \frac{\left(A_{1}-B_{1}\right)\left(A_{2}-B_{2}\right)}{\left(1-B_{1}\right)\left(1-B_{2}\right)} .
$$

After a short computation, we have

$$
\left(I^{m}(\lambda, l) h(z)\right)^{\prime}=\frac{1}{n} \int_{0}^{1}\left(\varphi_{1} * \varphi_{2}\right)(s z) s^{\frac{1}{n}-1} d s .
$$

Using Lemma 1.2, one obtains

$$
\operatorname{Re}\left[\left(I^{m}(\lambda, l) h(z)\right)^{\prime}\right]>2 \sigma_{3}-1+\left(1-\sigma_{3}\right) \cdot{ }_{2} F_{1}\left(1,1 ; \frac{1}{n}+1 ; \frac{1}{2}\right)
$$

thus the desired result follows at once.

Theorem 3.3. Let $-1 \leq B_{j}<A_{j} \leq 1, j=1,2$. If the functions $f_{j} \in$ $\mathcal{R}_{\lambda}^{m}\left(\eta ; A_{j}, B_{j}\right), \eta \geq 0, j=1,2$, then the function $\psi \in \mathcal{A}$ defined by 


$$
I^{m}(\lambda, l) \psi(z)=\int_{0}^{z}\left(\left(I^{m}(\lambda, l) f_{1}\right)^{\prime} *\left(I^{m}(\lambda, l) f_{2}\right)^{\prime}\right)(s) d s
$$

belongs to the class $\mathcal{R}_{\lambda}^{m}(\eta ; 1-2 \delta,-1)$ where

$$
\delta=\left\{\begin{array}{ll}
1-4 \frac{\left(A_{1}-B_{1}\right)\left(A_{2}-B_{2}\right)}{\left(1-B_{1}\right)\left(1-B_{2}\right)}\left[1-\frac{1}{2} \cdot{ }_{2} F_{1}\left(1,1, \frac{1}{n \eta}+1 ; \frac{1}{2}\right)\right], & \eta>0 \\
1-2 \frac{\left(A_{1}-B_{1}\right)\left(A_{2}-B_{2}\right)}{\left(1-B_{1}\right)\left(1-B_{2}\right)}, & \eta=0
\end{array} .\right.
$$

The bound $\delta$ is the best possible when $B_{1}=B_{2}=-1$.

\section{Proof. Letting}

$$
g_{j}(z):=\left(I^{m}(\lambda, l) f_{j}(z)\right)^{\prime}+\eta z\left(I^{m}(\lambda, l) f_{j}(z)\right)^{\prime \prime}, \quad \eta>0
$$

we note that $g_{j} \in \mathcal{P}\left(\sigma_{j}\right), j=1,2$ where $\sigma_{j}=\frac{1-A_{j}}{1-B_{j}}$ since $f_{j} \in \mathcal{R}_{\lambda}^{m}\left(\eta ; A_{j}, B_{j}\right)$.

One obtains that $\left(g_{1} * g_{2}\right) \in \mathcal{P}\left(\sigma_{3}\right)$ where $\sigma_{3}$ is given by (3.7).

From (3.11) we get

$$
\left(I^{m}(\lambda, l) f_{j}(z)\right)^{\prime}=\frac{1}{n \eta} z^{-\frac{1}{n \eta}} \int_{0}^{z} g_{j}(s) s^{\frac{1}{n \eta}-1} d s,
$$

thus by (3.9) and (3.12) after a short computation we have

$$
\begin{aligned}
\left(I^{m}(\lambda, l) \psi(z)\right)^{\prime} & =\left(\left(I^{m}(\lambda, l) f_{1}\right)^{\prime} *\left(I^{m}(\lambda, l) f_{2}\right)^{\prime}\right)(z) \\
& =\left(\frac{1}{n \eta} z^{-\frac{1}{n \eta}} \int_{0}^{z} g_{1}(s) s^{\frac{1}{n \eta}-1} d s\right) *\left(\frac{1}{n \eta} z^{-\frac{1}{n \eta}} \int_{0}^{z} g_{2}(s) s^{\frac{1}{n \eta}-1} d s\right) \\
& =\frac{1}{n \eta} \int_{0}^{1} u^{\frac{1}{n \eta}-1} v(u z) d u
\end{aligned}
$$

where

$$
\begin{aligned}
v(z) & =\left(I^{m}(\lambda, l) \psi(z)\right)^{\prime}+\eta z\left(I^{m}(\lambda, l) \psi(z)\right)^{\prime \prime} \\
& =\frac{1}{n \eta} \int_{0}^{1} u^{\frac{1}{n \eta}-1}\left(g_{1} * g_{2}\right)(z) d u .
\end{aligned}
$$

From Lemma 1.2 and (3.13) we obtain

$$
\operatorname{Re}(v(z)) \geq 2 \sigma_{3}-1+\left(1-\sigma_{3}\right){ }_{2} F_{1}\left(1,1, \frac{1}{n \eta}+1 ; \frac{1}{2}\right)=\delta .
$$

For the case $\eta=0$ the proof is simple and thus we omit the involved details. 
Theorem 3.4. Let $-1 \leq B<A \leq 1$. If $f \in \mathcal{R}_{\lambda}^{m}(\eta ; A, B)$ and $\varphi \in K$, then $f * \varphi \in \mathcal{R}_{\lambda}^{m}(\eta ; A, B)$.

Proof. It is well known that $\varphi \in K \Rightarrow \operatorname{Re}\left(\frac{\varphi(z)}{z}\right)>\frac{1}{2}, \quad(z \in U)$. Setting $h(z)=\left(I^{m}(\lambda, l) f(z)\right)^{\prime}+\eta z\left(I^{m}(\lambda, l) f(z)\right)^{\prime \prime}, g(z)=\frac{\varphi(z)}{z}$ and using convolution properties, one obtains

$$
\left(I^{m}(\lambda, l)(f * g)(z)\right)^{\prime}+\eta z\left(I^{m}(\lambda, l)(f * \varphi)(z)\right)^{\prime \prime}=(h * \varphi)(z) .
$$

Since $h$ is subordinate to the convex univalent function $(1+A z) /(1+B z)$ in $U$, our theorem is an immediate consequence of Lemma 1.4.

Theorem 3.5. Let $-1 \leq B_{j}<A_{j} \leq 1, j=1,2$ such that

$$
\frac{\left(A_{1}-B_{1}\right)\left(A_{2}-B_{2}\right)}{\left(1-B_{1}\right)\left(1-B_{2}\right)}<\frac{3}{4\left\{1+2\left[\frac{1}{2} \cdot{ }_{2} F_{1}\left(1,1 ; \frac{1}{n}+1 ; \frac{1}{2}\right)-1\right]^{2}\right\}}
$$

If the functions $f_{j} \in \mathcal{R}_{\lambda}^{m}\left(0 ; A_{j}, B_{j}\right)$, then the function $h$ defined by (3.1) satisfies the differential subordination

$$
\frac{z\left(I^{m}(\lambda, l) h(z)\right)^{\prime}}{I^{m}(\lambda, l) h(z)} \prec \frac{1+z}{1-z} .
$$

Proof. One obtains

$$
\begin{gathered}
\operatorname{Re}\left[\left(I^{m}(\lambda, l) h(z)\right)^{\prime}+z\left(I^{m}(\lambda, l) h(z)\right)^{\prime \prime}\right]= \\
=\operatorname{Re}\left[\left(I^{m}(\lambda, l) f_{1}(z)\right)^{\prime} *\left(I^{m}(\lambda, l) f_{2}(z)\right)^{\prime}\right]>1-2 \frac{\left(A_{1}-B_{1}\right)\left(A_{2}-B_{2}\right)}{\left(1-B_{1}\right)\left(1-B_{2}\right)} .
\end{gathered}
$$

From Theorem 3.2 we deduce that

$$
\begin{aligned}
& \operatorname{Re}\left(I^{m}(\lambda, l) h(z)\right)^{\prime} \\
> & 1-4 \frac{\left(A_{1}-B_{1}\right)\left(A_{2}-B_{2}\right)}{\left(1-B_{1}\right)\left(1-B_{2}\right)}\left[1-\frac{1}{2}{ }_{2} F_{1}\left(1,1 ; \frac{1}{n}+1 ; \frac{1}{2}\right)\right] .
\end{aligned}
$$

From (3.16) and Lemma 1.1 for $\gamma=1, B=-1$ and

$$
A=-1+8 \frac{\left(A_{1}-B_{1}\right)\left(A_{2}-B_{2}\right)}{\left(1-B_{1}\right)\left(1-B_{2}\right)}\left[1-\frac{1}{2} \cdot{ }_{2} F_{1}\left(1,1 ; \frac{1}{n}+1 ; \frac{1}{2}\right)\right]
$$

one obtains 
(3.17) $\operatorname{Re}(g(z))>1-8 \frac{\left(A_{1}-B_{1}\right)\left(A_{2}-B_{2}\right)}{\left(1-B_{1}\right)\left(1-B_{2}\right)}\left[\frac{1}{2}{ }_{2} F_{1}\left(1,1 ; \frac{1}{n}+1 ; \frac{1}{2}\right)-1\right]^{2}$

where

$$
g(z)=\frac{I^{m}(\lambda, l) h(z)}{z} .
$$

Letting

$$
\varphi(z):=\frac{z\left(I^{m}(\lambda, l) h(z)\right)^{\prime}}{I^{m}(\lambda, l) h(z)}, \quad(z \in U)
$$

we have

$$
\begin{aligned}
\left(I^{m}(\lambda, l) h(z)\right)^{\prime}+z\left(I^{m}(\lambda, l) h(z)\right)^{\prime \prime} & =g(z)\left[\varphi^{2}(z)+z \varphi^{\prime}(z)\right] \\
& =\psi\left(\varphi(z), z \varphi^{\prime}(z) ; z\right), \quad(z \in U) .
\end{aligned}
$$

It follows that

$$
\operatorname{Re}\left(\psi\left(\varphi(z), z \varphi^{\prime}(z) ; z\right)\right)>1-2 \frac{\left(A_{1}-B_{1}\right)\left(A_{2}-B_{2}\right)}{\left(1-B_{1}\right)\left(1-B_{2}\right)} .
$$

For all real numbers $\rho, \sigma, \sigma \leq-\frac{1}{2}\left(1+\rho^{2}\right)$ we have

$$
\begin{aligned}
& \quad \operatorname{Re}\{\psi(i \rho, \sigma ; z)\}=\operatorname{Re}\left\{g(z)\left(\sigma-\rho^{2}\right)\right\} \leq-\frac{1}{2}\left(1+3 \rho^{2}\right) \operatorname{Re} g(z) \\
& \leq-\frac{1}{2} \operatorname{Re} g(z) \leq 1-2 \frac{\left(A_{1}-B_{1}\right)\left(A_{2}-B_{2}\right)}{\left(1-B_{1}\right)\left(1-B_{2}\right)} .
\end{aligned}
$$

Thus, by an application of Lemma 1.5 we conclude that $\operatorname{Re} \varphi(z)>0$.

Remark 3.4. Taking $m=0, l=0, n=1, A_{j}=1-2 \alpha\left(0 \leq \alpha_{j}<1\right)$ and $B_{j}=-1$ for $j=1,2$ in Theorem 3.5 we get the corresponding results obtained by Lashin [4]. Similarly for $n=1, l=0$ we get the results obtained by Patel [8].

\section{REFERENCES}

1. F. M. Al-Oboudi, On univalent functions defined by a generalized Salagean operator, Int. J. Math. Math. Sci., 27 (2004), 1429-1436.

2. N. E. Cho and H. M. Srivastava, Argument estimates of certain analytic functions defined by a class of multiplier transformations, Math. Comput. Modelling, 37(1-2) (2003), 39-49.

3. N. E. Cho and T. H. Kim, Multiplier transformations and strongly close-to-convex functions, Bull. Korean Math. Soc., 40(3) (2003), 399-410. 
4. A. Y. Lashin, Some convolution properties of analytic functions, Appl. Math. Lett., 18 (2005), 135-138.

5. T. H. MacGregor, Functions whose derivative has a positive real part, Trans. Amer. Math. Soc., 104 (1962), 532-537.

6. S. S. Miller and P. T. Mocanu, Differential Subordination, Theory and Applications, Pure and Applied Mathematics, Marcel Dekker Inc., 2000.

7. D. Z. Pashkouleva, The starlikeness and spiral-convexity of certain subclasses of analytic functions, in: Current Topics in Analytic Function Theory, H. M. Srivastava, S. Owa (eds.), World Scientific Publishing Company, Singapore, New Jersey, London and Hong Kong, 1992.

8. J. Patel, Inclusion relations and convolution properties of certain subclasses of analytic functions defined by generalized Salagean operator, Bull. Belg. Math. Soc. Simon Stevin, 15(I) (2008), 33-47.

9. J. Patel, N. E. Cho and H. M. Srivastava, Certain subclasses of multivalent functions associated with a family of linear operators, Math. Comput. Modelling, 43 (2006), 320-338.

10. S. Ponnusamy, Differential subordination and starlike functions, Complex Variables Theory Appl., 19 (1992), 185-194.

11. G. S. Salagean, Subclasses of univalent functions, Complex Analysis-Fifth RomanianFinnish Seminar, Part 1 (Bucharest, 1981), Lecture Notes in Math., 1013, Springer, Berlin, 362-372.

12. R. Singh and S. Singh, Convolution properties of a class of starlike functions, Proc. Amer. Math. Soc., 108 (1989), 145-152.

13. J. Stankiewicz and Z. Stankiewicz, Some applications of the Hadamard convolution in the theory of functions, Ann. Univ. Mariae Curie-Sklodowska Sect. A, 40 (1986), 251-265.

14. B. A. Uralegaddi and C. Somanatha, Certain classes of univalent functions, Current topics in analytic function theory, 371-374, World Sci. Publishing, River Edge, N.J., (1992).

15. E. T. Whittaker and G. N. Watson, A course of Modern Analysis: An Introduction to the General Theory of Infinite Processes and of Analytic Functions; With an Account of the Principle Transcendental Functions, Fourth edition, Cambridge Univ. Press, Cambridge, 1927.

\author{
Adriana Cătaş \\ Department of Mathematics, \\ University of Oradea, \\ 1 University Street, \\ 410087 Oradea, \\ Romania \\ E-mail: acatas@gmail.com
}

